\title{
Gênero e sexualidade na formação de enfermeiros no ensino superior público brasileiro: estudo documental
}

\author{
Gender and sexuality in the training of nurses in brazilian public higher education: a documentary \\ study
}

\section{Género y sexualidad en la formación de enfermeras en la educación superior pública brasileña: un estudio documental}

\section{RESUMO}

Objetivo: Analisar o conteúdo das ementas de disciplinas que abordam gênero e sexualidade nos cursos de graduação em enfermagem. Método: Pesquisa documental com abordagem mista. Os dados foram coletados com instrumento de elaboração própria, processados no software IRaMuTeQ, apresentados em quadros, dendograma, categorias temáticas e analisados interpretativamente. Resultados: O conteúdo das ementas focalizou-se na saúde da mulher especificamente nos aspectos reprodutivos; políticas, diretrizes e parâmetros curriculares; histórica da sexualidade; práticas pedagógicas sobre gênero e sexualidade; saúde sexual e reprodutiva; conceito de gênero e intersecionalidades; gênero como categoria analítica e marcadores sociais da diferença; masculinidade e saúde do homem. Conclusão: Lacunas na estruturação dos currículos e abordagem de temáticas relativas a gênero e sexualidade contribuem para fragmentação dos processos formativos e práticas assistenciais.

Descritores: Identidade de Gênero; Sexualidade; Enfermagem; Educação Superior; Currículo.

\section{ABSTRACT}

Objective: To analyze the syllabuses of disciplines that address gender and sexuality in undergraduate nursing courses. Method: Documentary research with a mixed approach. Data were collected with an instrument of its own elaboration, processed in the IRaMuTeQ software, presented in tables, dendrograms, thematic categories and analyzed interpretively. Results: The content of the syllabuses of disciplines was focused on women's health, specifically on reproductive aspects; curriculum policies, guidelines and parameters; history of sexuality; pedagogical practices on gender and sexuality; sexual and reproductive health; concept of gender and intersectionalities; gender as an analytical category and social markers of difference; masculinity and men's health. Conclusion: Gaps in the structuring of curricula and addressing issues related to gender and sexuality contribute to the fragmentation of training processes and care practices. Descriptors: Gender Identity; Sexuality; Nursing; Education, Higher; Curriculum.

\section{RESUMEN}

Objetivo: Analizar el contenido de los planes de estudio de las disciplinas que abordan género y sexualidad en los cursos de enfermería. Método: Investigación documental con enfoque mixto. Los datos fueron recolectados con un instrumento de elaboración propia, procesados en el software IRaMuTeQ, presentados en tablas, dendrogramas, categorías temáticas y analizados interpretativamente. Resultados: El contenido de los planes de estudio se centró en la salud de la mujer, específicamente en los aspectos reproductivos; políticas, lineamientos y parámetros curriculares; historia de la sexualidad; prácticas pedagógicas sobre género y sexualidad; salud sexual y reproductiva; concepto de género e interseccionalidades; el género como categoría analítica y marcadores sociales de diferencia; masculinidad y salud de los hombres. Conclusión: Las brechas en la estructuración de los planes de estudio y el abordaje de temas relacionados con el género y la sexualidad contribuyen a la fragmentación de los procesos de formación y las prácticas de cuidado.

Descriptores: Identidad de Género; Sexualidad; Enfermería; Educación Superior; Curriculum.

\section{Ana Cristina Santos de Lima ${ }^{1}$ \\ 0000-0002-9211-5328}

Maria Juscinaide Henrique Alves ${ }^{1}$ D 0000-0002-5547-6247

Emanuelly Vieira Pereira ${ }^{1}$ (D) 0000-0003-1457-6281

Antonia Priscila Pereira ${ }^{1}$ 0000-0001-7581-1463

Grayce Alencar Albuquerque ${ }^{1}$ (D) 0000-0002-8726-0619

\section{Jameson Moreira Belém ${ }^{1}$ \\ 0000-0003-1903-3446}

${ }^{1}$ Universidade Regional do Cariri.

Autor correspondente:

Jameson Moreira Belém

E-mail: jam.ex@hotmail.com

\section{Como citar este artigo:}

Lima ACS, Alves MJH, Pereira EV, et al. Gênero e sexualidade na formação de enfermeiros no ensino superior público brasileiro: estudo documental. Revista de Enfermagem do Centro-Oeste Mineiro. 2021;11:e3877. [Access Available in: . DOI http://doi.org/10.19175/recom.v11i0.3 $\underline{877}$ 


\section{INTRODUÇÃO}

A enfermagem como ciência humanística busca desenvolver cuidados voltados à prevenção, promoção, cura e reabilitação de indivíduos e coletividades, precisando dispor de profissionais capacitados para abranger o ser humano em suas dimensões biopsicossocioespirituais ${ }^{(1)}$

Nessa perspectiva, as Diretrizes Curriculares Nacionais (DCNs) dos cursos de graduação em enfermagem apontam a necessidade de formação generalista, humanista, crítica e reflexiva ao definirem o perfil do discente com capacidade de atuar com senso de responsabilidade, comprometimento com a cidadania e promoção da saúde ${ }^{(2)}$.

Esses aspectos formativos devem contemplar as ciências biológicas e da saúde; humanas e sociais; e da enfermagem, sendo que, conteúdos essenciais devem estar relacionados ao processo saúde-doença dos usuários, famílias e comunidades, integrados à realidade epidemiológica e profissional para proporcionar integralidade das ações de cuidado ${ }^{(2)}$.

Apesar de existirem documentos norteadores que orientam a prática pedagógica nos cursos de graduação, essas orientações nem sempre contemplam todos os aspectos essenciais à formação do enfermeiro. Estudos com acadêmicos de enfermagem mostram que assuntos relativos a gênero e sexualidade não são, suficientemente, explorados e, quando as discussões ocorrem, são abordadas de forma superficial e resumida, por meio de aulas expositivas pontuais e palestras ${ }^{(1,3-4)}$.

Estudo com docentes de enfermagem apontou fragilidades na formação acadêmica relativos à sexualidade, ausência de preparo e dificuldades para abordagem e ensino dessas temáticas ${ }^{(3)}$.

Estudos com enfermeiros evidenciaram que o aprendizado sobre sexualidade ocorre via estereótipos de gênero na educação recebida na infância, adolescência, formação e prática profissional em diferentes cenários culturais (família, escola, universidade, serviços de saúde), personagens, roteiros interpessoais e intrapsíquicos, socialmente construídos, que definem posturas, atitudes e resultam em dificuldades quanto à inserção do tema como objeto de cuidado, à medida que as práticas profissionais são desenvolvidas fragmentadas, centradas em visão patologizante, com forte orientação biomédica, desconsiderando a abordagem sociocultural da sexualidade $\mathrm{e}^{(5-7)}$.

A discussão e reflexão acerca desses temas na formação do enfermeiro permite aos docentes romper com a cultura do silêncio, ao transpor proibições, tabus e preconceitos; e configuram possibilidade de instrumentalização dos estudantes para lidarem com diversas questões que podem suscitar no cotidiano do cuidado de enfermagem, realizando-o de forma segura, sem constrangimentos ou juízos de valor ${ }^{(4,8)}$.

A compreensão da sexualidade como elemento que precisa ser medicalizado, reforça o modelo biomédico. Em contraponto, quando ausente na dimensão do ser humano cuidado e, igualmente, na dimensão do enfermeiro cuidador, reforça o modelo assexualizado do cuidado(6). Desse modo, a abordagem pontual ou lacunas na formação do enfermeiro, acerca das temáticas gênero e sexualidade faz com que profissionais, por preconceito, falta de conhecimento e necessidade de impor valores, tornem a assistência enviesada ${ }^{(4,8)}$.

Estudos conduzidos, no Brasil, sobre gênero e sexualidade evidenciaram lacunas, na formação do enfermeiro e/ou dificuldades de abordagem dessas temáticas na prática profissional ${ }^{(1,3-9)}$. Desse modo, neste estudo, objetivou-se analisar o conteúdo das ementas de disciplinas que abordam gênero e sexualidade nos cursos de graduação em enfermagem, das Instituições de Ensino Superior (IES) públicas brasileiras.

\section{MÉTODO}

\section{Tipo de estudo}

Pesquisa documental desenvolvida a partir de dados de domínio público disponibilizados online que utilizou o software Interface de $R$ pour $L$ Analyses Multidimensionnelles de Textes $L$ de Questionnaires (IRaMuTeQ) como ferramenta de apoio ao processamento dos dados, sendo caracterizada como de abordagem mista à medida que permite a quantificação de variáveis essencialmente qualitativas originadas de textos, subsidiando a integração de análises quantitativas e qualitativas sobre determinado objeto de estudo.

\section{Período/população/critérios de seleção/definição da amostra}

A coleta de dados iniciou-se com busca realizada, entre agosto e outubro de 2018, de forma pareada, para conferir rigor metodológico 
de cursos de graduação em enfermagem, ofertados na modalidade presencial por IES públicas cadastradas no sistema eletrônico do ministério da educação E-mec, implementado em 2007, para (re)credenciamento, autorização e reconhecimento dos cursos das IES no Brasil.

Na primeira etapa, obtiveram-se 146 cursos de graduação em enfermagem. Posteriormente, buscaram-se Projetos Político-Pedagógicos (PPP), matrizes curriculares e/ou fluxogramas dos cursos disponíveis nos sites das IES.

Para a seleção e inclusão dos documentos, utilizou-se como critério a presença da palavra gênero e/ou do radical sexual na nomenclatura das disciplinas. Foram identificadas 61 disciplinas em 42 cursos. Dessas, 39 possuíam a palavra gênero e 22 continham o radical sexual na nomenclatura.

Para a análise do componente curricular, buscaram-se ementas, por conterem informações detalhadas sobre o ensino das temáticas, objeto do estudo. Apenas 23 disciplinas possuíam ementas disponíveis online na íntegra, sendo necessário contato, por meio de e-mail institucional das IES para solicitar as 38 indisponíveis ou incompletas. Aguardou-se um prazo de 15 dias para retorno, obtendo-se 13 ementas adicionais. Desse modo, compuseram amostra final ementas de 36 disciplinas ministradas em cursos de graduação em enfermagem de 26 IES públicas.

\section{Instrumento de coleta de dados}

Utilizou-se instrumento de elaboração própria para a coleta de dados contendo, na parte I, dados de caracterização da IES: Unidade de Federação (UF), região, categoria administrativa (municipal, estadual ou federal), organização acadêmica (faculdade, centro universitário, instituto federal ou universidade) e campus.

$\mathrm{Na}$ parte II, obtiveram-se dados relacionados ao curso de graduação: grau (bacharelado ou licenciatura), carga horária mínima e vagas anuais autorizadas. Na parte III, contemplaram-se dados relacionados às disciplinas: modalidade de oferta (optativa ou obrigatória), carga horária (teórica e/ou prática), ementa (descrição, objetivo(s) e conteúdo programático). Em função da diversidade de formatos e da não-uniformidade de conteúdo das ementas, optou-se por não incluir informações relativas às metodologias de ensino e avaliação.

\section{Organização dos dados}

As informações referentes ao conteúdo das ementas, compuseram o corpus e foram organizadas no programa Libre Office Writer, versão 5.3, seguindo orientações específicas para a construção do banco de dados. Adotou-se um sistema de codificação para as variáveis: região de localização da universidade (REG_1=Nordeste, REG_2=Norte, REG_3=Centro-oeste, REG_4=Sul e REG_5=Sudeste), Estados de localização das universidades (EST_01 e, assim, sucessivamente, para as 27 UF), grau dos cursos (GRA_1=Bacharelado e GRA_2=Licenciatura) e modalidade de oferta da disciplina (MOD_1=optativa e MOD_2=obrigatória). As ementas foram codificadas e numeradas, sequencialmente, à medida que os dados eram coletados (Exemplo: EME_01).

\section{Tratamento e análise dos dados}

Para tratamento dos dados referentes à IES, curso de graduação e oferta de disciplinas, utilizouse estatística descritiva (valores mínimo e máximo) e medidas de tendência central (média e moda).

O processamento dos dados qualitativos ocorreu, por meio do software Interface de $R$ pour $L$ Analyses Multidimensionnelles de Textes $L$ de Questionnaires(IRaMuTeQ), versão 0.7 alfa 2, que ancora-se no ambiente estatístico do Programa R, possibilitando diferentes análises sobre corpus textuais como a lexicográfica (cálculo de frequência de palavras) e multivariada (classificação hierárquica descendente, análises de similitude e nuvem de palavras).

Entre as formas de análise, utilizou-se a Classificação Hierárquica Descendente $(\mathrm{CDH})$, que divide o corpus em classes, agrupa as palavras de acordo com a maior associação entre elas e apresenta o percentual de representação no corpus estudado, para auxiliar na identificação da estrutura representada pelas ementas das disciplinas.

Os dados foram apresentados, descritivamente, em categorias temáticas e analisados de modo interpretativo, discutidos em conformidade à literatura pertinente.

\section{Aspectos éticos}

Esse tipo de estudo não necessita de apreciação ética. 


\section{RESULTADOS}

No Quadro 1, observam-se dados relativos à distribuição por localização e caracterização das IES.

Quadro 1 - Perfil das IES públicas com cursos de enfermagem

\begin{tabular}{|c|c|c|c|c|c|}
\hline \multirow[t]{2}{*}{ Região } & \multirow[t]{2}{*}{ Universidades } & \multicolumn{2}{|c|}{ Categoria administrativa } & \multirow[t]{2}{*}{ Estados } & \multirow[t]{2}{*}{ Campus } \\
\hline & & Federal & Estadual & & \\
\hline Nordeste & 13 & 09 & 04 & $\begin{array}{l}03 \text { Bahia } \\
03 \text { Ceará } \\
02 \text { Pernambuco } \\
02 \text { Rio Grande do Norte } \\
01 \text { Paraíba } \\
01 \text { Piauí } \\
01 \text { Sergipe }\end{array}$ & 16 \\
\hline Sudeste & 05 & 02 & 03 & $\begin{array}{l}02 \text { Minas Gerais } \\
02 \text { Rio de Janeiro } \\
01 \text { São Paulo }\end{array}$ & 06 \\
\hline Sul & 05 & 03 & 02 & $\begin{array}{l}02 \text { Paraná } \\
02 \text { Santa Catarina } \\
01 \text { Rio Grande do Sul }\end{array}$ & 05 \\
\hline Centro-oeste & 02 & 01 & 01 & $\begin{array}{l}01 \text { Goiás } \\
01 \text { Mato Grosso }\end{array}$ & 02 \\
\hline Norte & 01 & 01 & - & 01 Tocantins & 01 \\
\hline Total & 26 & 16 & 10 & 16 & 30 \\
\hline
\end{tabular}

Fonte: Dados da pesquisa.

Observou-se maior número de universidades federais $(n=16)$ que ofertam disciplinas relativas a gênero e/ou sexualidade com maior concentração no Nordeste $(n=13)$ e predomínio de cursos com grau bacharelado(n=29). As vagas anuais autorizadas para oferta, por curso, variaram entre 26 e 120 vagas, com moda 80 ( $n=08$ ) e média 65,7. A carga horária total dos cursos variou de 3.262 horas a 4.890 horas, com moda $4050(n=03)$ e média 4325,033 .

No Quadro 2, observam-se informações relativas às disciplinas e a sua distribuição por região.

Quadro 2 - Caracterização das disciplinas e sua distribuição espacial

\begin{tabular}{|c|c|c|c|c|c|c|}
\hline \multirow{2}{*}{ Região } & \multirow{2}{*}{ UF } & \multirow{2}{*}{ Palavra e/ou radical } & \multirow{2}{*}{ Modalidade } & \multicolumn{3}{|c|}{ Carga horária (h/a) } \\
\hline & & & & Teórica & Prática & Total \\
\hline \multirow{17}{*}{ Nordeste } & Bahia & Gênero & Optativa & 51 & - & 51 \\
\hline & Bahia & Gênero & Optativa & 60 & - & 60 \\
\hline & Bahia & Gênero & Optativa & 60 & - & 60 \\
\hline & Ceará & Sexual & Obrigatória & 100 & 110 & 210 \\
\hline & Ceará & Sexual & Obrigatória & 92 & 164 & 256 \\
\hline & Ceará & Gênero/Sexual & Optativa & 72 & - & 72 \\
\hline & Ceará & Gênero/Sexual & Optativa & 72 & - & 72 \\
\hline & Pernambuco & Gênero & Obrigatória & 120 & 120 & 240 \\
\hline & Pernambuco & Gênero & Obrigatória & 30 & - & 30 \\
\hline & Paraíba & Sexual & Optativa & 45 & - & 45 \\
\hline & Piauí & Gênero & Optativa & 60 & - & 60 \\
\hline & Rio Grande do Norte & Gênero & Obrigatória & 30 & - & 30 \\
\hline & Rio Grande do Norte & Gênero/Sexual & Optativa & 60 & - & 60 \\
\hline & Rio Grande do Norte & Gênero & Obrigatória & 30 & - & 30 \\
\hline & Rio Grande do Norte & Gênero & Obrigatória & 30 & - & 30 \\
\hline & Rio Grande do Norte & Gênero & Optativa & 45 & - & 45 \\
\hline & Sergipe & Sexual & Optativa & 45 & - & 45 \\
\hline Norte & Tocantins & Sexual & Obrigatória & 105 & 90 & 195 \\
\hline \multirow{2}{*}{$\begin{array}{l}\text { Centro- } \\
\text { oeste }\end{array}$} & Goiás & Gênero/ Sexual & Optativa & 60 & - & 60 \\
\hline & Mato Grosso & Sexual & Obrigatória & 96 & 208 & 304 \\
\hline
\end{tabular}


Quadro 2 - Caracterização das disciplinas e sua distribuição espacial

\begin{tabular}{|c|c|c|c|c|c|c|}
\hline \multirow{2}{*}{ Região } & \multirow{2}{*}{ UF } & \multirow{2}{*}{ Palavra e/ou radical } & \multirow{2}{*}{ Modalidade } & \multicolumn{3}{|c|}{ Carga horária (h/a) } \\
\hline & & & & Teórica & Prática & Total \\
\hline \multirow{7}{*}{ Sul } & Paraná & Gênero & Obrigatória & 96 & 127 & 223 \\
\hline & Paraná & Gênero & Optativa & 30 & - & 30 \\
\hline & Santa Catarina & Gênero & Optativa & 36 & - & 36 \\
\hline & Santa Catarina & Sexual & Optativa & 36 & - & 36 \\
\hline & Santa Catarina & Gênero/Sexual & Obrigatória & 36 & - & 36 \\
\hline & Rio Grande do Sul & Gênero/Sexual & Optativa & 60 & - & 60 \\
\hline & Rio Grande do Sul & Gênero/Sexual & Optativa & 30 & & 30 \\
\hline \multirow{9}{*}{ Sudeste } & Minas Gerais & Sexual & Optativa & 30 & - & 30 \\
\hline & Minas Gerais & Gênero & Optativa & 45 & - & 45 \\
\hline & Minas Gerais & Sexual & Optativa & 30 & 30 & 60 \\
\hline & Rio de Janeiro & Sexual & Optativa & 30 & - & 30 \\
\hline & São Paulo & Sexual & Optativa & 15 & - & 15 \\
\hline & São Paulo & Gênero & Optativa & 45 & - & 45 \\
\hline & São Paulo & Sexual & Optativa & 30 & - & 30 \\
\hline & São Paulo & Sexual & Optativa & 30 & - & 30 \\
\hline & São Paulo & Sexual & Obrigatória & 30 & - & 30 \\
\hline
\end{tabular}

Fonte: Dados da pesquisa.

Abreviaturas: $\mathrm{h} / \mathrm{a}=$ horas-aula, $\mathrm{UF}=$ Unidades Federativas.

Em relação à carga horária total das disciplinas ( $n=36)$, observou-se variação de $15 \mathrm{~h} / \mathrm{a}$ à 304h/a, moda 30h/a ( $n=11)$ e média de 75,58h/a. Entre as optativas ( $n=24)$, variou de $15 \mathrm{~h} / \mathrm{a}$ à $72 \mathrm{~h} / \mathrm{a}$, com moda 60h/a ( $n=7$ ) e média de 46,12h/a, tendo apenas uma disciplina teórico-prática. Entre as obrigatórias ( $n=12$ ) variou de 30h/a à 304h/a, com moda $30 \mathrm{~h} / \mathrm{a}(\mathrm{n}=5)$ e média de $134,5 \mathrm{~h} / \mathrm{a}$, sendo que seis apresentavam carga horária teórica e seis teórico-prática.

O IRaMuTeQ processou o corpus textual e reconheceu 36 unidades de texto correspondendo à quantidade de ementas obtidas, 174 segmentos de texto, 5776 ocorrências de palavras, com 1319 formas, das quais 1021 eram formas ativas (palavras analisáveis indicadoras de sentido, de acordo com o que as ementas apresentavam) e 66 formas suplementares. O número de formas ativas com frequência foi de $>=3: 312$, a média das formas por segmento foi de 33.195402 e o número de clusters foi oito, que constituem agrupamentos baseados em frequência. Obteve-se índice de $100 \%$ de aproveitamento dos textos.

Na Figura 1, verifica-se dendograma que ilustra a $\mathrm{CDH}$ resultante do processamento do corpus textual.

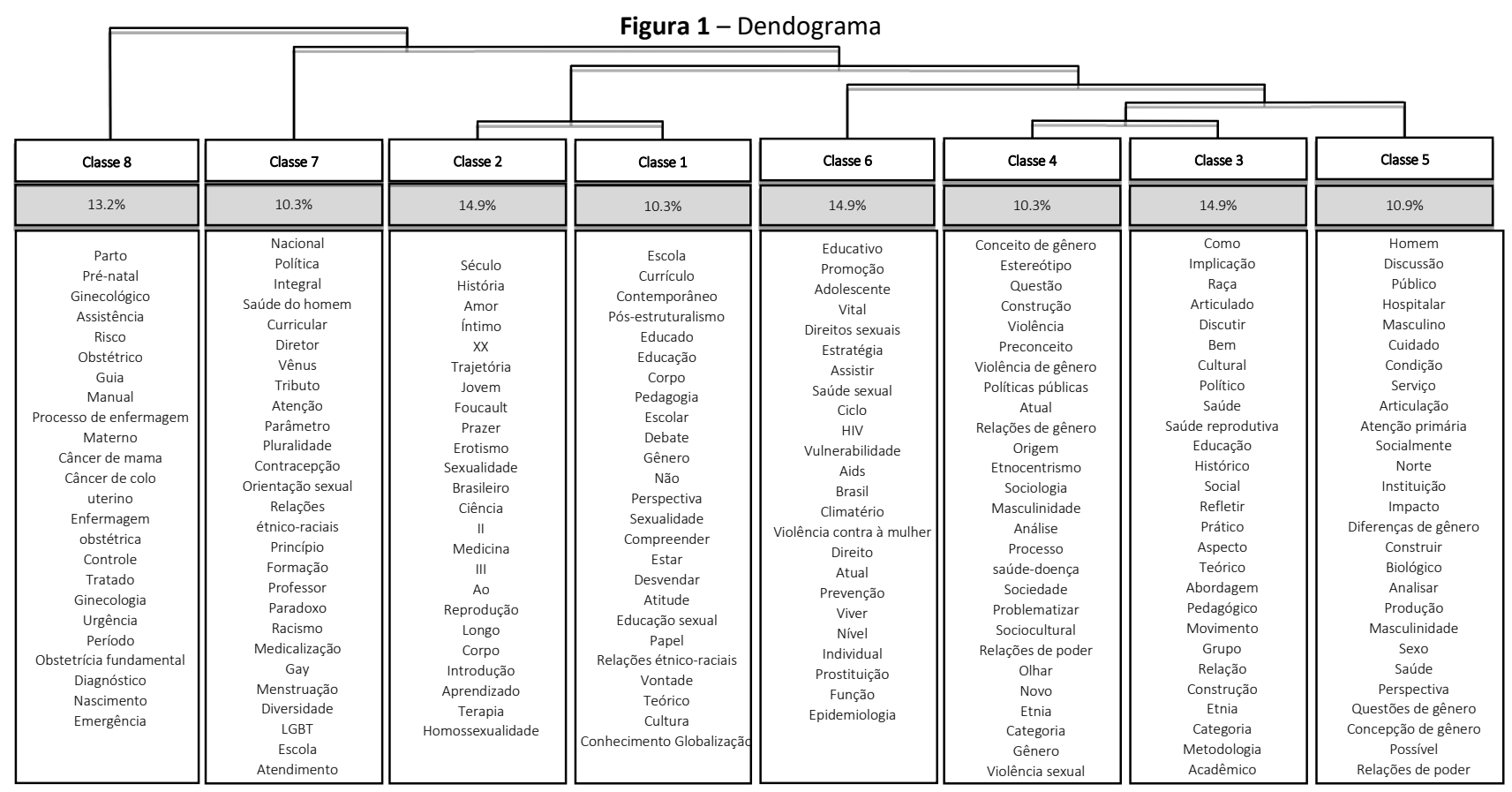

Fonte: Dados da pesquisa. 
O dendrograma apresenta oito classes, que constituem classificações baseadas em algoritmo. $\mathrm{Na}$ distribuição, o corpus textual ramifica-se, inicialmente, em subconjuntos de palavras/expressões-chave. Da primeira ramificação (classe 8 ) originam-se as demais classes.
No Quadro 3, apresentam-se as categorias temáticas construídas com base nas classes, que apresentaram percentagens maiores de ocorrência dos segmentos de texto que continham a palavra na classe em relação a sua ocorrência no corpus; e respectivos trechos das ementas evidenciando palavras representativas.

Quadro 3 - Categorias temáticas elaboradas a partir das classes

\begin{tabular}{|c|c|}
\hline \multirow[t]{2}{*}{$\begin{array}{l}\text { Aspectos históricos na abordagem } \\
\text { da sexualidade (Classe } 2 \text { - 14.9\%) }\end{array}$} & $\begin{array}{l}\text { O aprendizado da sexualidade, reprodução e trajetórias sociais de jovens brasileiros; } \\
\text { sexualidade e medicina: a revolução do século XX; as técnicas do corpo; a construção da } \\
\text { diferença sexual na medicina: uma ciência da diferença (Ementa 06, Nordeste, Ceará, } \\
\text { Bacharelado, Optativa, Escore: 178.07). }\end{array}$ \\
\hline & $\begin{array}{l}\text { A luta contra a sífilis no Brasil: da passagem do século aos anos 40; histórias íntimas; } \\
\text { sexualidade e erotismo na história do Brasil; mitos e tabus da sexualidade humana: } \\
\text { subsídios ao trabalho em educação sexual (Ementa 07, Nordeste, Ceará, Bacharelado, } \\
\text { Optativa, Escore: } 135.61 \text { ). }\end{array}$ \\
\hline \multirow[t]{2}{*}{$\begin{array}{l}\text { Gênero como categoria analítica e } \\
\text { marcadores sociais da diferença } \\
\text { (Classe } 3-14,9 \%)\end{array}$} & $\begin{array}{l}\text { Problematizar a inclusão do construto gênero na elaboração de políticas públicas de saúde } \\
\text { e de educação, assim como nas práticas associadas a essas políticas; apreender a } \\
\text { construção social, cultural e histórica da sexualidade humana e da saúde reprodutiva } \\
\text { articulando às categorias gênero, raça, etnia e classe social (Ementa 06, Nordeste, Ceará, } \\
\text { Bacharelado, Optativa, Escore: } 228.63 \text { ). }\end{array}$ \\
\hline & $\begin{array}{l}\text { Refletir sobre as implicações da articulação entre gênero, etnia, raça e geração para saúde } \\
\text { de pessoas e/ou grupos sociais; discutir a contribuição do feminismo, movimento negro e } \\
\text { movimento LGBT }{ }^{1} \text { para a formulação de políticas de saúde (Ementa 01, Nordeste, Bahia, } \\
\text { Bacharelado, Optativa, Escore: 171.33). }\end{array}$ \\
\hline \multirow[t]{2}{*}{$\begin{array}{l}\text { Saúde sexual e reprodutiva: } \\
\text { desafios atuais, práticas educativas } \\
\text { e assistenciais na perspectiva do } \\
\text { ciclo vital (Classe } 6-14,9 \% \text { ) }\end{array}$} & $\begin{array}{l}\text { Práticas educativas e prevenção de } H I V^{2} / \text { Aids }^{3} \text { : lições aprendidas e desafios atuais; os } \\
\text { conceitos de vulnerabilidade e adesão na saúde coletiva; a sexualidade de adolescentes } \\
\text { vivendo com HIV: direitos e desafios para o cuidado (Ementa 36, Sudeste, São Paulo, } \\
\text { Bacharelado, Optativa, Escore: } 146.20 \text { ). }\end{array}$ \\
\hline & $\begin{array}{l}\text { Discutir a contracepção de emergência, o aborto inseguro, atenção à violência contra à } \\
\text { mulher e temas afins, na perspectiva dos direitos sexuais e reprodutivos e sua relação com } \\
\text { a enfermagem; direitos humanos e saúde sexual e reprodutiva: histórias e conceitos; } \\
\text { práticas educativas na promoção dos direitos humanos, direitos sexuais e reprodutivos } \\
\text { (Ementa 31, Sudeste, Rio de Janeiro, Bacharelado, Optativa, Escore: 124.04). }\end{array}$ \\
\hline \multirow[t]{2}{*}{$\begin{array}{l}\text { Saúde da mulher com enfoque nos } \\
\text { aspectos reprodutivos (Classe } 8 \text { - } \\
13.2 \% \text { ) }\end{array}$} & $\begin{array}{l}\text { Assistência de enfermagem à saúde sexual e reprodutiva; processo de cuidar no período } \\
\text { pré-natal, parto e puerpério; processo de cuidar nas situações de urgências e emergências } \\
\text { obstétricas; cuidados de enfermagem no pré-operatório, transoperatório e pós-operatório } \\
\text { ginecológico e obstétrico; planejamento familiar (Ementa 05, Nordeste, Ceará, } \\
\text { Bacharelado, Obrigatória, Escore: } 339.48 \text { ). }\end{array}$ \\
\hline & $\begin{array}{l}\text { Habilitar para a realização das etapas do processo de enfermagem, histórico, exame físico } \\
\text { ginecológico e obstétrico; estabelecimento do diagnóstico, metas e prescrição de } \\
\text { enfermagem na assistência integral à saúde da mulher; enfermagem no ciclo gravídico } \\
\text { puerperal; enfermagem obstétrica e ginecológica (Ementa 08, Nordeste, Pernambuco, } \\
\text { Bacharelado, Obrigatória, Escore: 181.77). }\end{array}$ \\
\hline
\end{tabular}

${ }^{1}$ LGBT = Lésbicas, Gays, Bissexuais e Transexuais; ${ }^{2}$ HIV = Human Immunodeficiency Vírus; ${ }^{3}$ Aids = Acquired Immunodeficiency Syndrome.

Fonte: Dados da pesquisa.

No Quadro 4, apresentam-se as categorias temáticas construídas com base nas classes, que apresentaram percentagens menores de ocorrência dos segmentos de texto que continham a palavra na classe, em relação a sua ocorrência no corpus; e respectivos trechos das ementas, evidenciando palavras representativas. 
Quadro 4 - Categorias temáticas elaboradas a partir das classes

Práticas pedagógicas sobre gênero e sexualidade na escola (Classe $1-10,3 \%$ )

Conceito de gênero e suas interseccionalidades (Classe $4-10,3 \%)$

Masculinidade e saúde do homem (Classe 5-10,9\%)

Políticas, diretrizes e parâmetros curriculares

(Classe 7-10,3\%)

O corpo educado, gênero, sexualidade e educação: uma perspectiva pós-estruturalista. Corpo, gênero e sexualidade: um debate contemporâneo na educação. Corpos, gêneros e sexualidades: questões possíveis para o currículo escolar (Ementa 24, Sul, Rio Grande do Sul, Bacharelado, Disciplina Optativa, Escore: 234.01).

Compreender que a escola não apenas transmite saberes e conhecimentos, mas tem sido um dos locais centrais no processo de produção de determinado tipo de sujeito e de suas identidades; compreender o estudo das questões relativas ao gênero (Ementa 24, Sul, Rio Grande do Sul, Bacharelado, Disciplina Optativa, Escore: 78.83). O conceito de cultura, natureza e cultura; antropologia da saúde e da doença; etnocentrismo, estereótipo e preconceito; as origens e aplicações do conceito de gênero e classificação social; construção social da identidade e as marcas do gênero; diferenças sexuais (Ementa 06, Nordeste, Ceará, Bacharelado, Optativa, Escore: 148.74).

Elaborar um arcabouço de conhecimentos sobre temas como diversidade, etnia, gênero, sexualidade e orientação sexual; o conceito de cultura; heteronormatividade; conceito de gênero e sexualidade; etnocentrismo, estereótipo e preconceito; as origens e aplicações do conceito de gênero e classificação social (Ementa 07, Nordeste, Ceará, Bacharelado, Optativa, Escore: 134.48).

Discussão da condição do ser homem e do ser mulher como categoria construída histórica e, socialmente, a partir das relações de poder estabelecidas na sociedade; desconstrução da naturalização biológica das diferenças de gênero; a produção dos serviços de saúde, enfermagem e sua articulação com as questões de gênero (Ementa 12, Nordeste, Rio Grande do Norte, Bacharelado, Obrigatória, Escore: 216.66).

Concepção de gênero de homens usuários e profissionais de saúde de serviços de atenção primária à saúde e os possíveis impactos na saúde da população masculina; equidade de gênero e saúde da mulher; concepção de gênero, masculinidade e cuidados em saúde: estudo com profissionais de saúde da atenção primária à saúde; gênero e saúde: diálogos ibero-brasileiros (Ementa 12, Nordeste, Rio Grande do Norte, Bacharelado, Obrigatória, Escore: 170.97).

Gênero, sexualidade e saúde reprodutiva: parâmetros curriculares nacionais; apresentação dos temas transversais; ética; parâmetros curriculares nacionais; pluralidade cultural e orientação sexual; Política Nacional de Saúde Integral de Lésbicas, Gays, Bissexuais, Travestis e Transexuais (Ementa 06, Nordeste, Ceará, Bacharelado, Optativa, Escore: 340.19).

Fonte: Dados da pesquisa.

\section{Saúde da mulher com enfoque nos aspectos reprodutivos}

Essa classe aborda o processo de se cuidar a mulher, com ênfase nos aspectos ginecológicos, obstétricos, reprodutivos e assistenciais de enfermagem. As ementas centraram-se na obstetrícia básica e fundamental, perinatologia básica, saúde da mulher, enfermagem em saúde da mulher, enfermagem no ciclo gravídicopuerperal, enfermagem obstétrica e ginecológica, enfermagem materno-infantil.

Incorporaram o estudo e compreensão das patologias comuns associadas ao sistema reprodutor e impacto na vida da mulher; buscaram habilitar estudantes para a realização das etapas do processo de enfermagem, na assistência à saúde (histórico, exame físico ginecológico e obstétrico, diagnóstico, metas e prescrição de enfermagem), bem como o desenvolvimento de atitudes e habilidades necessárias ao cuidado qualificado com base nos preceitos legais, éticos e técnico-científicos.

Incluíram discussões no âmbito do processo de cuidar (atenção, assistência, cuidados e orientações na perspectiva do ciclo vital) na anticoncepção, contracepção de emergência, gestação de alto risco, pré-natal de baixo risco, parto, nascimento, puerpério, climatério, abortamento e cirurgias ginecológicas (com ênfase em qualidade de vida, bem-estar, acolhimento, classificação de risco, humanização, boas práticas, 
segurança do paciente, urgências e emergências obstétricas), promoção da saúde, prevenção, controle e vigilância das ISTs/Aids e dos cânceres ginecológicos e mamários, respostas emocionais no ciclo gravídico-puerperal, às doenças e ao adoecer.

Focalizaram na SAE à saúde sexual e reprodutiva, em ginecologia, pré-parto, alojamento conjunto $e$ às pessoas com ISTs/Aids/hepatites virais, no desenvolvimento de consulta de enfermagem em saúde da mulher e cuidados de enfermagem nos períodos pré, trans e pós operatórios gineco-obstétricos.

Utilizaram dispositivos para subsidiar a prática educativa-assistencial como guias diagnósticos, terapêuticos e de conduta (situações de risco de morte materna, patologias obstétricas, atenção efetiva na gravidez e parto, atenção à saúde do recém-nascido e práticas assistenciais em enfermagem obstétrica e ginecológica), manuais técnicos e de bolso (acolhimento e classificação de risco em obstetrícia, controle das ISTs, Atenção Integrada às Doenças Prevalentes na Infância, orientação em obstetrícia e ginecologia, atenção à mulher no climatério e menopausa), tratados (fisiologia humana, infectologia, ginecologia, obstetrícia e enfermagem obstétrica), diretrizes assistenciais e normas técnicas.

As discussões perpassaram princípios e diretrizes da política nacional de atenção integral à saúde da mulher, programa de humanização no pré-natal e nascimento, programação e avaliação da assistência de enfermagem à saúde sexual e reprodutiva na rede de atenção à saúde da mulher e nos serviços de Atenção Primária à Saúde (APS).

\section{Políticas, diretrizes e parâmetros curriculares}

Essa classe apresenta o arcabouço normativo e legal que norteia as políticas públicas de saúde e educação com fundamento na perspectiva de gênero e sexualidade.

As ementas buscaram descrever princípios e diretrizes para a operacionalização da atenção integral à saúde de LGBTs, mulheres, homens e pessoas idosas. Resgataram o caráter histórico de combate às "doenças venéreas", reflexões sobre menstruação, contracepção, aborto e paradoxos da medicalização na perspectiva de gênero. As discussões voltadas à atualidade, incorporaram implicações bioéticas, no atendimento de saúde ao público LGBT, aspectos jurídicos no atendimento às vítimas de violência sexual, direitos humanos, sexuais e reprodutivos na atenção à saúde e sua relação com a enfermagem.

Trataram dos parâmetros curriculares contidos na Lei de Diretrizes e Bases (LDB) da educação nacional e abordagem de temas transversais voltados às relações étnico-raciais, racismo, preconceito, discriminação racial e suas manifestações na sociedade, diversidade, orientação sexual, diferenças de gênero, discriminação sexual e de gênero e pluralidade cultural na escola. Para desenvolvimento de orientações e ações para educação, nessa perspectiva, apontaram práticas pedagógicas e formação de professores em gênero e sexualidade, pautadas no respeito à diversidade.

\section{Aspectos históricos na abordagem da sexualidade}

Essa classe apresenta a história da construção da sexualidade como dispositivo legitimado pelo saber médico-científico, fundamentado na noção da diferença sexual, sobretudo a partir da revolução do século XX e de vertentes da filosofia, antropologia cultural e da saúde, interpretação das culturas com ênfase na afro-brasileira, pensamentos foucaultianos, freudianos e da psicanálise.

As ementas direcionaram-se à introdução de temáticas voltadas ao aprendizado da sexualidade, homossexualidade, reprodução e trajetórias sociais, sobretudo de jovens brasileiros, bem como, problemas de importância para as ciências da saúde. Ainda, voltaram-se à compreensão, reflexão crítica, debates contemporâneos no contexto da educação, concepções sobre sexualidade feminina, estudos e abordagens de gênero e do (homo)erotismo.

Nas ementas, as discussões históricas da sexualidade perpassaram por diferentes épocas e ethos constituídos, à medida que enfatizaram um resgate das histórias íntimas e transformação da intimidade, reprodução, amor, uso dos prazeres sexuais, prazer estético, práticas, perversões, mitos, papéis sexuais, uso e culto ao corpo sexualizado, análise do corpo sob a perspectiva das ciências biológicas, (homo)erotismo e luta contra a sífilis, sobretudo no contexto histórico brasileiro.

Algumas discussões centraram-se em diferentes noções de sexualidade feminina no climatério, terceira idade, conjugalidade, gestação e processo de hospitalização. 


\section{Práticas pedagógicas sobre gênero e sexualidade na escola}

Nesta classe, as ementas buscaram desvendar, debater, discutir e compreender questões relativas ao corpo, gênero e sexualidade, na educação, em uma perspectiva crítica, teórica, analítica, conceitual, socioantropológica, cultural e pós-estruturalista, situando esses fenômenos em sua historicidade para apreensão na contemporaneidade.

Esses debates estavam centrados em discussões relativas ao corpo educado e sexualizado, na perspectiva de desvendar o aprendizado e as atitudes em relação à sexualidade. Prevalece a representação da escola como espaço privilegiado para a inclusão de reflexões e debates sobre educação sexual, nesse cenário, e no âmbito familiar.

A formação de professores e estruturação do currículo escolar, por meio de pedagogias da sexualidade, são apontados como elementos que devem oportunizar a abordagem de questões relativas à identidade, igualdade, diferenças, diversidade, orientação sexual, relações de gênero, étnico-raciais e de nacionalidade em interface com aspectos éticos, políticos, midiáticos, religiosos, históricos, tecnológicos e, principalmente, com a saúde e o papel do enfermeiro.

\section{Saúde sexual e reprodutiva: desafios atuais, práticas educativas e assistenciais na perspectiva do ciclo vital}

Essa classe aborda a saúde sexual e reprodutiva e sua interface com identidades, poder e direitos, sobretudo relacionados aos direitos humanos, sexuais e reprodutivos, políticas públicas e de atenção à saúde da mulher e gênero. Apresentaram um panorama e epidemiologia das questões de saúde reprodutiva, no Brasil, aspectos políticos, sociais, culturais, éticos e étnico-raciais.

Teceram um resgate histórico e abordaram os conceitos de saúde materna, saúde sexual e reprodutiva, saúde da mulher, direitos sexuais e reprodutivos, educação, vulnerabilidade e adesão na saúde coletiva e no contexto da Aids, sexualidade, diversidade e orientação sexual.

Abordaram aspectos e práticas educativas relacionadas à sexualidade, saúde sexual e reprodutiva nas fases do ciclo vital como estratégias para a promoção dos direitos humanos, sexuais e reprodutivos, na perspectiva da APS com ênfase na Estratégia Saúde da Família e sua relação com a enfermagem.
Centralizaram discussões em temas éticos da atualidade como: aborto, violência contra à mulher por parceiro íntimo, cirurgia plástica, sexo, práticas educativas e preventivas em sexualidade voltadas às ISTs/Aids, drogas e violência sobretudo para adolescentes, prostituição incluindo a infantil, expressões da sexualidade, religião, morte (eutanásia, distanásia, ortotanásia e mistanásia), apontados como desafios atuais, transculturais e para o Sistema Único de Saúde (SUS).

Discutiram práticas assistenciais e de enfermagem voltadas à prevenção, promoção e recuperação da saúde sexual e reprodutiva nos diferentes ciclos da vida, na perspectiva dos direitos sexuais e reprodutivos, por vezes com enfoque na APS e na assistência integral à saúde da mulher, enfatizando as vítimas de violência.

Direcionaram-se a compreender o processo saúde-doença das mulheres, características da sexualidade feminina, do casal, condutas e respostas sexuais humanas e o ciclo reprodutivo da mulher perpassando por repercussões, intercorrências e complicações.

Pautaram especificidades do cenário brasileiro e discussões em enfoques atuais e multidisciplinares, perspectivas não medicalizantes, sob os princípios de gênero e da humanização, nos modos de ser e viver a corporeidade, o gênero e a sexualidade, bem como o desenvolvimento de atividades individuais e coletivas, avaliação de fatores de vulnerabilidade, promoção da saúde sexual no ciclo vital, realidades e possibilidades de assistir em enfermagem, consultas e Sistematização da Assistência de Enfermagem (SAE) nas áreas de saúde da mulher, enfermagem obstétrica e enfermagem maternoneonatal.

Abordaram aspectos relacionados à iniciação sexual de adolescentes, educação para a saúde, prevenção e controle do uso de álcool e drogas, na vida e no trabalho, violências de gênero a mulheres, LGBTs, crianças e adolescentes, HIV/Aids, sífilis, hepatites e outras ISTs no Brasil (evolução epidemiológica, políticas públicas, estratégias de controle, modelos e práticas de prevenção, abordagem coletiva e individual de enfrentamento desses agravos), bem como a sexualidade, direitos e desafios para o cuidado de adolescentes, vivendo com HIV.

\section{Conceito de gênero e suas interseccionalidades}

As ementas dessa classe abordaram as origens, aplicação, antecedentes históricos e 
filosóficos relacionados à evolução do conceito de gênero, apresentando-o como construção sóciohistórica e categoria analítica útil para compreender, problematizar e abordar as relações de poder, questões transnacionais e transculturais das relações de gênero na sociedade, saúde e enfermagem.

A partir de marcos teóricos, estudos e pesquisas da sociologia com contribuição do feminismo, as ementas apresentaram arcabouço de conhecimento para análise conceitual e crítica de questões relativas à saúde, corpo, gênero, sexualidade, diversidade, orientação sexual, geração, raça, etnia e suas interseccionalidades, como formas de classificação social presentes, histórica e, culturalmente, na sociedade, bem como compreender suas implicações e intervenções no processo saúde-doença, concepção e gerenciamento das políticas públicas de saúde.

As ementas buscam discutir processos sociais de construção da feminilidade e masculinidade, diferenças (etnocentrismo, estereótipo e preconceito), identidades, heteronormatividade, novas identidades sexuais e, inclusive, a construção histórica da enfermagem sob a ótica de gênero.

Abordaram, sob olhar de gênero, discussões presentes na sociedade contemporânea relacionadas à vida e processo saúde-doença das mulheres, formas de opressão e violência (gênero e sexual), masculinidade, novos contextos de cidadania sexual e afetiva individuais e familiares, transversalidade de gênero nas políticas públicas e suas repercussões na atenção à saúde dos indivíduos e coletividades e construção da identidade profissional do enfermeiro.

\section{Gênero como categoria analítica e marcadores sociais da diferença}

As ementas buscaram problematizar, analisar, estudar, refletir, discutir, desvendar, destacar, compreender, articular e incluir, em uma perspectiva interdisciplinar e pós-estruturalista, por meio de abordagens teóricas, conceituais e históricas, o gênero (construto, conceito e relações) como categoria analítica e sua articulação (interseccionalidades e transversalidade) com sexualidade, diversidade sexual, corpo, raça, cor, etnia, classe social e geração como construções históricas, culturais, sociais, econômicas, políticas e discursivas, bem como da saúde reprodutiva, educação e práxis pedagógica.

Nas ementas, a articulação entre gênero e marcadores sociais da diferença é apontada como produtora de implicações para a saúde de pessoas e/ou grupos, práticas socioculturais e avaliação dos serviços de saúde. Essas discussões subsidiam nas ementas a análise de aspectos demográficos e epidemiológicos, práticas de saúde, educativas e de enfermagem, em face das especificidades culturais de diferentes grupos (com destaque para mulheres e LGBTS), relacionando-as às condições de vida da população, bem como, às práticas assistenciais, gerenciais e educativas em saúde nas comunidades, instituições e serviços de saúde.

Apontaram, ainda, práticas pedagógicas em APS, tecnologias para abordagem do indivíduo, família, comunidade e educação sexual, fundamentadas em metodologias participativas feministas aplicadas ao cuidado de enfermagem e saúde e na perspectiva da diversidade para a formação de profissionais críticos com relação a comportamentos, abordagens excludentes e preconceituosas.

Essas práticas de saúde, educação e enfermagem envolveram a produção e aplicação de conhecimentos específicos no campo da saúde da mulher, considerando a construção do saber científico e da identidade feminina na sociedade. Além disso, discutiram a contribuição de movimentos (feminismo, negros e LGBT) para a formulação de políticas de saúde e educação.

\section{Masculinidade e saúde do homem}

Essa classe aborda o conceito e diferenciação entre saúde e doença e o estudo das inter-relações que conformam a complexidade da natureza humana dos sujeitos sociais e das relações humanas no contexto das ciências sociais e da saúde.

As discussões centralizaram-se na condição de gênero (ser homem e ser mulher) enquanto categoria construída histórica e socialmente, a partir das relações de poder estabelecidas na sociedade, na análise de fatores socioculturais e de gênero que influenciam nas diferenças de comportamento entre homens e mulheres e desconstrução da naturalização biológica das diferenças de gênero em uma perspectiva pósestruturalista, das relações sociais e dos estudos culturais.

Abordaram as relações e articulações entre questões de gênero, saúde, medicina, corpo sexo e 
sociedade que envolvem homens e mulheres, especialmente da sexualidade masculina, masculinidade, classe e posições sociais. Apresentaram um olhar sobre os corpos na história, novas configurações de corpos na atualidade, dominação masculina, pedagogias do corpo feminino, suas articulações com a produção do corpo saudável e desafios bioéticos.

Destacaram a concepção de gênero e possíveis impactos na saúde da população masculina, produção dos serviços, atuação dos profissionais e o papel da APS para atendimento integral das necessidades de saúde de homens, bem como a relação entre gênero, masculinidades e cuidado à saúde nesse nível de atenção.

Evidenciaram a articulação da enfermagem com questões de gênero, sexualidade e saúde, apresentando um olhar sobre a influência da categoria gênero na prestação do cuidado de enfermagem e suas interfaces entre as experiências no nível individual e coletivo nos serviços básicos de saúde, rede hospitalar, ambulatorial, de saúde pública e instituições de ensino.

\section{DISCUSSÃO}

A análise evidenciou a pouca quantidade de disciplinas que abordam gênero e sexualidade de forma específica, na grade curricular dos cursos de graduação em enfermagem das IES públicas brasileiras.

As escolas e universidades constituem espaços de formação de sujeitos com perspectiva crítica sobre as relações sociais, capazes de produzir movimentos de transformação na sociedade $^{(10)}$. Considerando a determinação social, na saúde, aponta-se a formação de profissionais qualificados para compreender o processo saúdedoença e as necessidades de saúde de distintos segmentos sociais.

Nesse sentido, destaca-se a categoria profissional de enfermagem que constitui a maior força de trabalho na área da saúde, no Brasil, e atua em todos os níveis de atenção, lidando, diretamente, com demandas de saúde da população(11). Ressalta-se que a formação profissional com perspectiva crítico-reflexiva sobre relações sociais marcadas pela dominação, exclusão e discriminação prepara o estudante para atender às necessidades de populações vulneráveis e reduzir disparidades de saúde ${ }^{(10,12)}$.

Enfatiza-se a necessidade de inserção, padronização e abordagem de temáticas transversais, entre as quais se destacam conteúdos relativos a gênero e sexualidade, como identidade, diversidade, igualdade e diferença, nas práticas pedagógicas e currículos de enfermagem para fornecer cuidados integrais ${ }^{(13-15)}$.

A falta de compreensão de enfermeiros sobre gênero e sexualidade pode afetar, negativamente, o tipo e a qualidade do atendimento à medida que não se fornecem cuidados culturalmente sensíveis em todas as fases do ciclo vital ${ }^{(16)}$. Esse contexto formativo fragilizado se agrava pela ausência de qualificação profissional continuada como reflexo de uma formação que prioriza a quantidade de conteúdos ministrados. Embora conteúdos relativos a gênero e sexualidade estejam sendo discutidos, o ensino ocorre de forma superficial ${ }^{(17)}$.

Estratégias de ensino e programas de treinamento para cuidados de saúde voltados a essas temáticas podem desenvolver conhecimentos, habilidades e atitudes nos acadêmicos, docentes e profissionais; promover competência cultural, sensibilidade e reduzir dificuldades de abordagem nos atendimentos ${ }^{112-}$ 13,18-21).

Entre as disciplinas analisadas, neste estudo, observou-se a não uniformidade em relação aos conteúdos ministrados, modalidade, oferta da disciplina e carga-horária. Estudos evidenciam necessidade de revisar currículos de enfermagem quanto às lacunas na abordagem de temáticas inerentes a gênero e sexualidade, sobretudo à saúde de populações vulneráveis, entre as quais se destaca a $\mathrm{LGBT}^{(13,15,21-23)}$.

Esse processo não se limita a examinar conteúdos programáticos, procedimentos clínicos, atividades pedagógicas e avaliativas, mas a incorporar valores sociais que reconheçam a diversidade, respeito e igualdade, integrem e reconheçam necessidades de saúde para desenvolver práticas livres de sexismo, homofobia, preconceitos e estereótipos ${ }^{(22-23)}$.

Quando se analisa o conteúdo das disciplinas, deste estudo, em sua totalidade observa-se que contemplam diversidade de conteúdos e temáticas essenciais, embora concentrados na saúde sexual e reprodutiva, por vezes centralizados em aspectos biológicos, patológicos, técnico-assistenciais e acerca da mulher.

Essa visão biomédica, tecnicista e patologizante se materializa, nas práticas de enfermagem, no âmbito da sexualidade e demonstram pouca relação com a promoção da 
saúde, nos atendimentos e consultas onde evidenciam-se dificuldades em abordar a sexualidade dos usuários ${ }^{(4)}$. Além disso, menor procura pelos serviços de saúde dos usuários pode estar associada à discriminação e/ou despreparo dos profissionais para lidar com a diversidade sexual $\left.\right|^{(7)}$.

Durante a formação do enfermeiro, a abordagem da sexualidade nas disciplinas e conteúdos curriculares têm pouca relação com gênero, limitando-se a aspectos biológicos ou reprodutivos às vezes com viés patologizante, reducionismo à genitalidade e/ou neutralização dos corpos sexuados, assexualização do sujeito cuidado e da assistência prestada, ocultamento da temática e visão tecnicista do cuidado, desconsiderando determinantes de saúde e relações sociais imbricadas no processo saúdedoença ${ }^{(8,24)}$.

O caráter de eventualidade e informalidade ao longo da formação constituem mecanismos geradores de insegurança, angústia, constrangimento, reações negativas ou indesejadas no cuidado de enfermagem que remetem ao despreparo para lidar com a sexualidade, principalmente se esta foge dos padrões heteronormativos ${ }^{(8-9)}$.

Em face dessa lacuna, enfermeiros, ao exercerem atividades assistenciais e docentes, acabam reproduzindo o modelo heteronormativo, que prevalece, durante sua formação acadêmica, consequentemente suas práticas de cuidado e/ou pedagógicas tendem a ser respaldadas em crenças e valores pessoais ${ }^{(9,18)}$.

Há discrepância de carga horária entre as disciplinas nos cursos analisados. Ressalta-se que disciplinas com carga-horária menores podem não fornecer reflexões e subsídios necessários para atuação na prática assistencial. A prevalência de disciplinas teóricas pode dificultar a compreensão do discente quanto à aplicabilidade prática e sua articulação com a assistência de enfermagem. Além disso, o caráter optativo da maioria das disciplinas dificulta a inserção dessas discussões nos processos formativos à medida que não é garantida a oferta da disciplina de forma contínua.

Desse modo, apontam-se como estratégias para reduzir vieses assistenciais a estruturação de modelos e práticas pedagógicas interdisciplinares capazes de desenvolver conhecimentos, habilidades e atitudes nos acadêmicos, para avaliar e reconhecer vulnerabilidades em saúde relacionadas ao modo de ser e viver a corporeidade, o gênero e a sexualidade, discutindo essas questões no contexto da educação e da saúde articuladas à vida cotidiana. Essa perspectiva pode ampliar o olhar dos profissionais para além da visão biomédica e, consequentemente, perceber os indivíduos/coletividades, suas histórias, vulnerabilidades, desejos, valores e crenças ${ }^{(7,18,20)}$.

As categorias gênero e sexualidade, como construções históricas, culturais e sociais, devem ser vistas de modo interseccional para compreender experiências, práticas e subjetividades de sujeitos sociais que ocorrem na coletividade. Essa abordagem, durante a formação deve possibilitar a reorientação das práticas profissionais às especificidades culturais dos grupos sociais, reflexão crítica sobre comportamentos, abordagens excludentes ou preconceituosas que influenciam na assistência ${ }^{(7)}$.

Nesse contexto, novos parâmetros curriculares devem emergir como estratégia programática para que cursos de enfermagem possam incorporar atividades teórico-práticas voltadas para a aquisição de competências clínicas e culturais acerca de gênero e sexualidade no processo de cuidar, com ênfase na integralidade e equidade da assistência, reorientando processos formativos, a criação, implementação e avaliação de políticas públicas, sociais e de saúde ${ }^{(1,12,20,24-25)}$.

Nesse sentido, deve-se oportunizar discussões sobre didática, estratégias de apoio educacional e ensino-aprendizagem para incorporar esses conteúdos nos currículos de enfermagem e saúde na graduação e pósgraduação(15,19,20,24,25). Estratégias podem ser utilizadas para promover maior integração dessas temáticas ao currículo, como: simulação, estudos de caso, planos de cuidados de enfermagem, cursos de capacitação, discussões, estudos independentes e cursos eletivos extracurriculares, parcerias com instituições, serviços de saúde e especialistas, grupos de estudo, pesquisas, aconselhamento acadêmico, educação interprofissional, workshops e consultorias ${ }^{(15,20,22-}$ 23,25).

Essas discussões não podem se restringir a momentos pontuais, mas apresentarem caráter permanente e integrado, que reforcem a necessidade de estruturação de programas, currículos mínimos e/ou disciplinas específicas voltadas ao ensino de gênero e sexualidade, no cuidado em saúde com estímulo contínuo para que as discussões sejam realizadas de modo transversal em outras disciplinas ao longo do curso ${ }^{(15,19-20,24)}$. 
Apesar das limitações operacionais associadas à indisponibilidade, baixo quantitativo de PPPs e ementas, déficit de devolutivas das solicitações desses documentos, acesso e funcionamento de sites institucionais comprometidos, o estudo aponta caminhos para revisar, atualizar, estruturar e operar mudanças curriculares que subsidiem a inclusão, consolidação de temas transversais e reorientação dos processos formativos e assistenciais.

\section{CONCLUSÃO}

Gênero e sexualidade constituem determinantes de saúde que devem ser abordados na formação profissional. Nos cursos de enfermagem das IES públicas brasileiras, observam-se lacunas na abordagem dessas temáticas, pouca quantidade de disciplinas, conteúdos não uniformes, irregularidade na oferta e cargas horárias diversificadas que contribuem para a fragmentação dos processos formativos e, consequentemente, das práticas assistenciais.

\section{REFERÊNCIAS}

1 - Figueiroa MN, Menezes MLN, Monteiro EMLM, Andrade ÂRL, Fraga DPF, Oliveira MV. A formação relacionada com a sexualidade humana na perceção de estudantes de enfermagem. Rev Enf Ref. 2017; 4(15):21-30. DOI: 10.12707/RIV17044

2 - Magnago C, Pierantoni CR. Nursing training and their approximation to the assumptions of the National Curriculum Guidelines and Primary Health Care. Ciênc Saúde Coletetiv 2019;25(1):15-24. DOI: $\underline{10.1590 / 1413-81232020251.28372019}$

3 - Rezende VA, Sobral OJ. As temáticas relativas à sexualidade humana na formação superior do profissional de Enfermagem. Revista Científica FacMais [citado em 7 ago 2020]; 5(1):25-39. Disponível em:

http://revistacientifica.facmais.com.br/wp-

content/uploads/2016/06/2-

\%20As\%20tem\%c3\%a1ticas\%20relativas\%20\%c3

\%a0\%20sexualidade\%20humana\%20na\%20forma \%c3\%a7\%c3\%a30\%20superior\%20do\%20profissio nal\%20de\%20Enfermagem.pdf

4 - Nogueira IS, Rodrigues DMR, Labegalini CMG, Lopes MCL, Baldissera VDA. Perception and formation of nursing academics regarding human sexuality. Rev Fund Care 2017;9(3):614-9. DOI: 10.9789/2175-5361.rpcfo.v9.5562
5 - Santos SMP, Freitas JLGS, Freitas MIF. The sexuality scripts constructed by nurses and an interface with attention of sexually transmited infection/HIV. Esc Anna Nery 2019;23(4):1-9. DOI: 10.1590/2177-9465-ean-2019-0078en

6 - Sehnem GD, Rodrigues RL, Lipinski JM, Vasquez MED, Schmidt A. (Des)preparo técnico-científico para o cuidado às travestis: Percepções de enfermeiras(os). Rev Enferm UFSM 2017;7(2):23647. DOI: $\underline{10.5902 / 2179769223649}$

7 - Belém JM, Alves MJH, Pereira EV, Moreira FTLS, Quirino GS, Albuquerque GA. Health care for lesbian, gay, bisexual, transvestite and transgender individuals in the family health strategy. Rev Baiana Enferm. 2018;32:1-13. DOI: 10.18471/rbe.v32.26475

8 - Sehnem GD, Ressel LB, Junges CF, Silva FM, Barreto CN. A sexualidade na formação acadêmica do enfermeiro. Esc Anna Nery 2013;17(1):90-6. DOI: $10.1590 /$ S1414-81452013000100013

9 - Andrade CAA, Lima Neto ER, Loureiro AR, Araújo EC. A diversidade sexual e as práticas pedagógicas do enfermeiro na docência. Rev Enferm UFPE 2017;11(11):1-2. DOI: 10.5205/19818963-v11i11a230727p\%25p-2017

10 - Welter T, Grossi MP. É possível ensinar gênero na escola? Análise de experiências de formação em gênero, sexualidade e diversidades em Santa Catarina. Rev Linhas 2018;19(39):123-45. DOI: $\underline{10.5965 / 1984723819392018123}$

11 - Silva MCN, Machado MH. Health and work system: Challenges for the Nursing in Brazil. Ciênc Saúde Coletiva 2020;25(1):7-13. DOI: 10.1590/1413-81232020251.27572019

12 - Strong KL, Folse VN. Assessing undergraduate nursing students knowledge, attitudes, and cultural competence in caring for lesbian, gay, bisexual, and transgender patients. J Nurs Educ. 2015;54(1):45-9. DOI: 10.1002/hon.2900070607

13 - Cappiello J, Coplon L, Carpenter H. systematic review of sexual and reproductive health care content in nursing curricula. J Obstet, Gynecol Neonatal Nurs. 2017;46(5):E157-E67. DOI: 10.1016/j.jogn.2017.04.132 
14 I Lima ACS, Alves MJH, Pereira EV, et al.

14 - Aaberg V. The state of sexuality education in baccalaureate nursing programs. Nurse Educ Today 2016; 44:14-9. DOI: 10.1016/i.nedt.2016.05.009

15 - McNiel PL, Elertson KM. Advocacy and awareness: Integrating LGBTQ health education into the prelicensure curriculum. I Nurs Educ. 2018;57(5):312-4. DOI: $10.3928 / 01484834-$ 20180420-12

16 - Carabez R, Scott M. 'Nurses don't deal with these issues': Nurses' role in advance care planning for lesbian, gay, bisexual and transgender patients. J Clin Nurs. 2016;25(23/24):3707-15. DOI: 10.1111/jocn.13336

17 - Cornelius JB, Enweana I, Alston CK, Baldwin DM. Examination of lesbian, gay, bisexual, and transgender health care content in North Carolina Schools of Nursing. J Nurs Educ. 2017;56(4):223-6. DOI: 10.3928/01484834-20170323-06

18 - Fennell R, Grant B. Discussing sexuality in health care: A systematic review. J Clin Nurs. 2019;28(17-18):3065-76.

DOI: 10.1111/jocn.14900

19 - McCann E, Brown M. The inclusion of LGBT+ health issues within undergraduate healthcare education and professional training programmes: A systematic review. Nurse Educ Today 2018;64:204-14. DOI: 10.1016/j.nedt.2018.02.028

20 - Sekoni AO, Gale NK, Manga-Atangana B, Bhadhuri A, Jolly K. The effects of educational curricula and training on LGBT-specific health issues for healthcare students and professionals: A mixed-method systematic review. J Int AIDS Soc. 2017;20(1):21624. DOI: 10.7448/IAS.20.1.21624

21 - Aslan F, Şahin NE, Emiroğlu ON. Turkish nurse educators knowledge regarding LGBT health and their level of homophobia: A descriptive-cross sectional study. Nurse Educ Today 2019;76:21621. DOI: $10.1016 /$ i.nedt.2019.02.014

22- Lim F, Brown D, Jones H. Lesbian, gay, bisexual, and transgender health: Fundamentals for nursing education. J Nurs Educ. 2013;52(4):198-203. DOI: http://doi.org/10.3928/01484834-20130311-02
23 - Unlu H, Beduk T, Duyan V. The attitudes of the undergraduate nursing students towards lesbian women and gay men. J Clin Nurs. 2016;25(2324):3697-706. DOI: $10.1111 /$ jocn.13347

24 - Yingling CT, Cotler K, Hughes TL. Building nurses' capacity to address health inequities: Incorporating lesbian, gay, bisexual and transgender health content in a family nurse practitioner programme. J Clin Nurs. 2017;26(1718):2807-17. DOI: $10.1111 /$ jocn.13707

25 - Walker K, Arbour M, Waryold J. Educational strategies to help students provide respectful sexual and reproductive health care for lesbian, gay, bisexual, and transgender persons. J Midwifery Womens Health 2016;61(6):737-43. DOI: 10.1111/jmwh.12506

Nota: Artigo extraído do Trabalho de Conclusão do Curso de Graduação em Enfermagem intitulado: "Ensino de gênero e sexualidade nos cursos de graduação em enfermagem: pesquisa documental"; apresentado à Universidade Regional do Cariri, 2018. Informamos que não houve financiamento por agência de fomento.

Recebido em: 28/06/2020

Aprovado em: 09/03/2021 